\title{
MODIFIED PYRAGAS METHOD FOR MULTIPLE SPATIAL LIMIT SETS AND CHAOS CONTROL IN MIMO CASCADE NONLINEAR SYSTEMS*
}

\author{
UDC ((681.511.2+531+534+517.93):124.1)
}

\author{
Biljana Samardžić ${ }^{1}$, Bojana Zlatković \\ ${ }^{1}$ University of Niš, Faculty of Science and Mathematics, Niš, Republic of Serbia \\ ${ }^{2}$ University of Niš, Faculty of Occupational Safety, Niš, Republic of Serbia
}

\begin{abstract}
The modified Pyragas method for the multiple spatial limit sets and chaos control in MIMO cascade nonlinear systems is presented in this paper. Also, an oscillatory and chaotic dynamic analysis of a specific MIMO3 cascade nonlinear system is performed before and after the application of control. Bifurcation diagrams and spatial phase portraits of uncontrolled and controlled MIMO3 systems are used for the purposes of analysis.
\end{abstract}

Key words: Modified Pyragas method, MIMO cascade nonlinear system, multiple spatial limit sets, chaos, bifurcation diagram, spatial phase portraits

\section{INTRODUCTION}

The Pyragas method, also known as the time-delayed feedback control method (TDFC), is applied to stabilize the unstable periodic orbits, [1,2]. In Figure 1 the block diagram of the Pyragas method is shown. In this diagram $y(t)$ represents the output variable, $\tau$ is a time delay, $K$ is a negative feedback gain, $p_{0}$ is a value at which the dynamical system has an unstable periodic orbit with a period $\tau$ and $p_{0}-K(y(t)-u(t-\tau))$ represents a new controlled input to system, [1].

In paper [3] a modified Pyragas method of spatial limit sets and chaos control in MIMO (multiple input - multiple output) cascade nonlinear systems was first introduced. In these systems the appearance of spatial limit sets and chaos is a consequence of signal propagation through space, i.e., through subsystems of MIMO cascade nonlinear systems. Also, in [3] the analogy between time delay of the original Pyragas method, [1], and

Received July 26, 2018

Corresponding author: Biljana Samardžić

Faculty of Science and Mathematics, Višegradska 33, 18000 Niš, Republic of Serbia

E-mail: biljana@pmf.ni.ac.rs

* Acknowledgement: The authors gratefully acknowledge the support from Ministry of Education and Science of the Republic of Serbia through projects ON171025 and 43014. 
spatial delay of the modified Pyragas method is made. Applying the modified Pyragas method, the dynamics of MIMO systems moves from the spatial limit set and chaos region to the spatial stability region. Firstly, based on spatial phase portraits diagrams, parameter values of uncontrolled MIMO cascade nonlinear systems for which spatial limit sets and chaos appear are obtained. After that, using selected values of the control parameter in the modified Pyragas method, the spatial limit sets and chaos are controlled. For MIMO cascade nonlinear systems with $n$ inputs and $n$ outputs, the spatial phase portraits of limit sets and chaos are shown in the phase space $\left(\mathbf{x}_{1}, \mathbf{x}_{2}, \ldots, \mathbf{x}_{\mathbf{n}}\right)$, where $\mathbf{x}_{\mathbf{i}}, i=\overline{1, n}$ is the vector of all $i$ th cascades outputs. Because of that in the same figure (spatial phase portrait), for the constant value of the variable parameter $l_{n}$, dynamics of all cascades (subsystems) is shown.

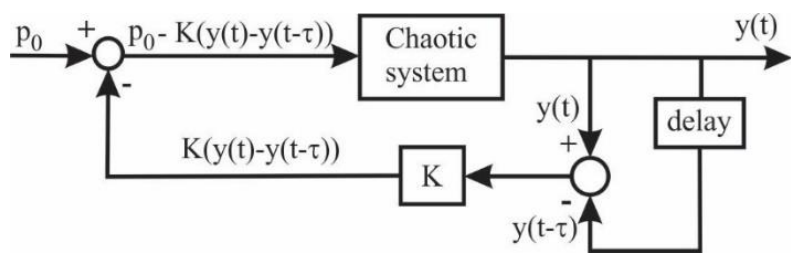

Fig. 1 Block diagram of the Pyragas control method

The modified Pyragas method for multiple spatial limit sets and chaos control is used in this paper. Multiple spatial limit sets consist of sets of mutually separated points in the phase plane. These points are situated in such a way so as to form approximately closed curves. In this paper, for the first time, bifurcation diagrams are used, [4-10], in the analysis of the applied modified Pyragas method of control. In the case of the MIMO system with a large number of cascades, monitoring dynamics of the whole system, using the bifurcation diagram, is based on monitoring of all outputs of the last cascade as a function of variable system parameter.

This paper is organized as follows. In Section 2, MIMO cascade nonlinear systems and the modified Pyragas method for multiple spatial limit sets and chaos control are presented. The simulation of bifurcation diagram and spatial phase portraits of uncontrolled MIMO3 cascade nonlinear system with 3 inputs and 3 outputs is given in Section 3. In the next Section, simulation results are presented after using the modified Pyragas method for multiple spatial limit sets and chaos control. The concluding remarks are presented in Section 5 and Matlab M-files for deriving bifurcation diagrams are given in Appendix.

\section{MiMO CASCAdE Nonlinear System AND ModifiEd PyRAGAS METHOD}

The MIMO cascade nonlinear system with $n$ inputs and $n$ outputs consists of a large number of nonlinear subsystems. These subsystems are of the same structure, Fig. 2, and each cascade is defined by the following set of equations: 


$$
\begin{aligned}
& x_{1}^{(i+1)}=f_{1}^{(i+1)}\left(x_{1}^{(i)}, \ldots, \quad x_{n}^{(i)}\right)=x_{2}^{(i)} \\
& x_{2}^{(i+1)}=f_{2}^{(i+1)}\left(x_{1}^{(i)}, \ldots, \quad x_{n}^{(i)}\right)=x_{3}^{(i)} \\
& \vdots \\
& x_{n-1}^{(i+1)}=f_{n-1}^{(i+1)}\left(x_{1}^{(i)}, \ldots, \quad x_{n}^{(i)}\right)=x_{n}^{(i)} \\
& x_{n}^{(i+1)}=f_{n}^{(i+1)}\left(x_{1}^{(i)}, \ldots, \quad x_{n}^{(i)}\right)= \\
& =-l_{1} x_{n}^{(i)}-l_{2} x_{n-1}^{(i)}-\ldots-l_{n} x_{1}^{(i)}, \quad i=\overline{0, k}
\end{aligned}
$$

where $l_{j}=l_{j}\left(\mathbf{x}^{(i)}\right), j=\overline{1, n}, i=\overline{0, k}$ are nonlinear functions whose argument is the input vector of each cascade $\mathbf{x}^{(i)}=\left[x_{1}^{(i)}, \ldots, x_{n}^{(i)}\right] \quad(i=\overline{0, k})$. This cascade system has in total $k+1$ cascades. Each cascade has $n$ inputs and $n$ outputs. The number $(i+\underline{1})$ in brackets is the ordinal of the cascade. The set of functions $f_{m}^{(i+1)}\left(x_{1}^{(i)}, \ldots, x_{n}^{(i)}\right), m=\overline{1, n}, i=\overline{0, k}$ is the same for each cascade. Functions $f_{m}^{(i+1)}\left(x_{1}^{(i)}, \ldots, x_{n}^{(i)}\right), m=\overline{1, n-1}, i=\overline{0, k}$ are linear, only the function $f_{n}^{(i+1)}\left(x_{1}^{(i)}, \ldots, x_{n}^{(i)}\right), i=\overline{0, k}$ is nonlinear. The input vector of the first cascade of the MIMO cascade system, Fig. 2, is $\mathbf{x}^{(0)}=\left[x_{1}^{(0)}, \ldots, x_{n}^{(0)}\right]$.

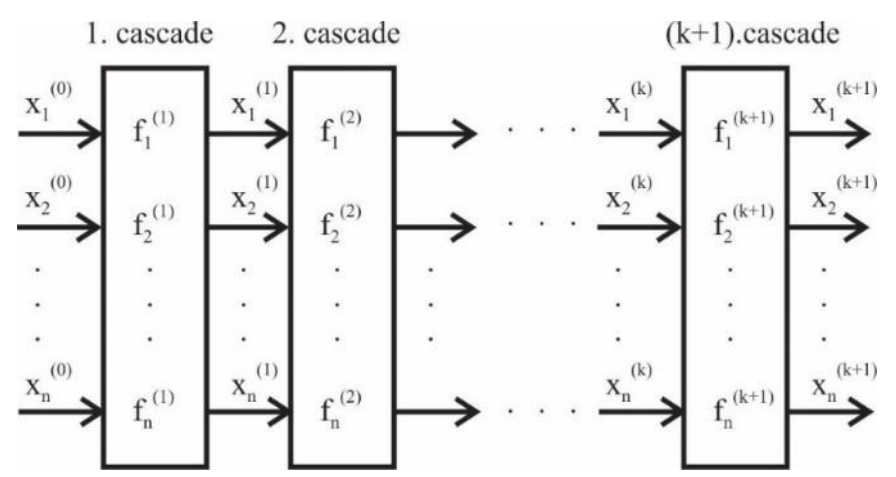

Fig. 2 The block scheme of MIMO cascade nonlinear system with $n$ inputs and $n$ outputs

For easier determination of existence or absence of limit sets in a MIMO cascade nonlinear system the approximation method is shown in $[3,11]$. Using this method the mutual position of the trajectory $l$ in parametric space is determined:

$$
l \Leftrightarrow \begin{gathered}
l_{1}=l_{1}\left(x^{(i)}\right) \\
l_{2}=l_{2}\left(x^{(i)}\right) \\
\vdots \\
l_{n}=l_{n}\left(x^{(i)}\right)
\end{gathered}
$$

and the stability region $S_{n}$ obtained using a linearized model of the system given by equations (1). It is possible to form multiple limit sets, if the curve $l$ intersects the region $S_{n}$ many times. Motion becomes more chaotic, the more the parametric curve $l$ approaches the peripheral part of the stability region. For the region $S_{n}$ the following relations hold, [3]: 


$$
\begin{aligned}
& \text { a) } S_{n} \in P_{n} \\
& \text { b) } O_{n} \in S_{n}
\end{aligned}
$$

where the region $P_{n}$ (hyper parallelepiped) is given with:

$$
P_{n} \Leftrightarrow\left|l_{i}\right| \leq\left(\begin{array}{c}
n \\
i
\end{array}\right), i=\overline{1, n}
$$

and the region $O_{n}$ (simplex) is:

$$
O_{n} \Leftrightarrow\left|l_{1}\right|+\left|l_{2}\right|+\ldots+\left|l_{n}\right| \leq 1
$$

Based on conditions (3), it can be concluded that the region $S_{n}$ is inside of the hyper parallelepiped $P_{n}$ and that the region $O_{n}$ is inside of the region $S_{n}$. The necessary conditions for the existence of multiple spatial limit sets mean that the curve $l$, (3), must intersect many times both regions $P_{n}$ and $O_{n}$.

The modified Pyragas method for spatial limit sets and chaos control is applied to a MIMO system with $n$ inputs and $n$ outputs and it is shown on Figure 3. Mathematical models of each controlled cascade are described with the following relations:

$$
\begin{aligned}
& y_{1}^{(i+1)}=f_{1}^{(i+1)}\left(y_{1}^{(i)}, \ldots, \quad y_{n}^{(i)}\right)=y_{2}^{(i)} \\
& y_{2}^{(i+1)}=f_{2}^{(i+1)}\left(y_{1}^{(i)}, \ldots, \quad y_{n}^{(i)}\right)=y_{3}^{(i)} \\
& \vdots \\
& y_{n-1}^{(i+1)}=f_{n-1}^{(i+1)}\left(y_{1}^{(i)}, \ldots, \quad y_{n}^{(i)}\right)=y_{n}^{(i)} \\
& y_{n}^{(i+1)}=f_{n}^{(i+1)}\left(y_{1}^{(i)}, \ldots, \quad y_{n}^{(i)}\right)-u^{(i)}= \\
& =-l_{1} y_{n}^{(i)}-l_{2} y_{n-1}^{(i)}-\ldots-l_{n} y_{1}^{(i)}-u^{(i)} \\
& u^{(i)}=K\left(y_{n}^{(i)}-y_{n-1}^{(i)}\right), i=\overline{0, k}
\end{aligned}
$$

where $l_{j}=l_{j}\left(\mathbf{y}^{(i)}\right), j=\overline{1, n}, i=\overline{0, k}$ are nonlinear functions whose argument is the input vector of each cascade $\mathbf{y}^{(i)}=\left[y_{1}^{(i)}, \ldots, \quad y_{n}^{(i)}\right], \quad i=\overline{0, k}$.

Inputs to the first cascade of the controlled MIMO cascade system (6) $\mathbf{y}^{(0)}=$ $\left[y_{1}^{(0)}, \ldots, y_{n}^{(0)}\right]$ and inputs to the first cascade of the uncontrolled MIMO cascade system $(1), \mathbf{x}^{(0)}=\left[x_{1}^{(0)}, \ldots, x_{n}^{(0)}\right]$, are the same, i.e., $\mathbf{y}^{(0)}=\mathbf{x}^{(0)}$.

In this case the difference $y(t)-y(t-\tau)$ from the Pyragas method, [1], is replaced with the difference of signals $y_{n}^{(i)}-y_{n}^{(i-1)}=y_{n}^{(i)}-y_{n-1}^{(i)}$, [3]. Thus, control of the $(i+1)$ th cascade is proportional to the difference of the $n$th and $(n-1)$ th input signals of the same cascade, $u^{(i)}=K\left(y_{n}^{(i)}-y_{n-1}^{(i)}\right), i=\overline{0, k} \cdot K$ is the control parameter. 
The analysis of the region where multiple spatial limit sets and chaos appear can be performed easilly using bifurcation diagrams. In the next sections this will be shown on the examples of uncontrolled and controlled MIMO3 systems.

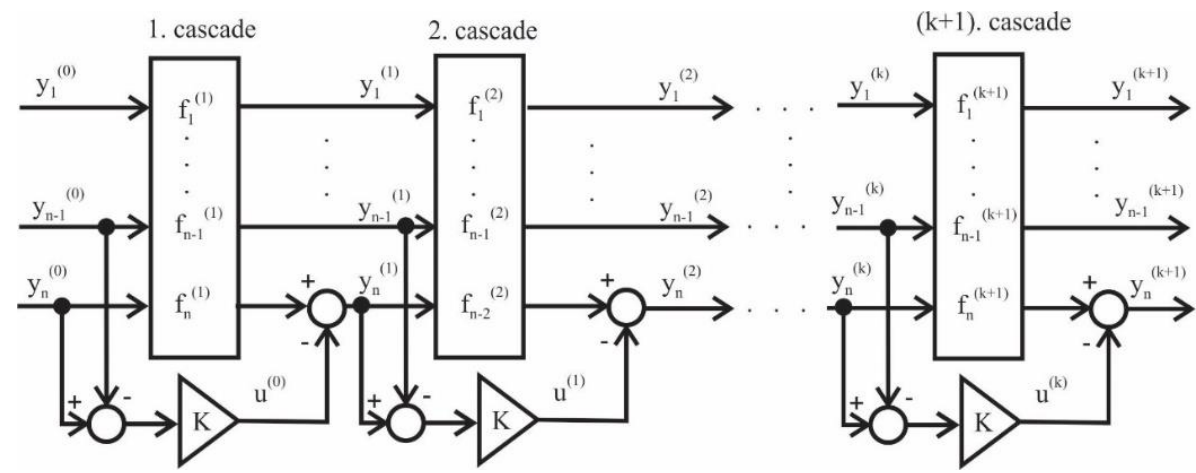

Fig. 3 Modified Pyragas method for control in MIMO cascade nonlinear systems with $n$ inputs - $n$ outputs

\section{EXAMPLE OF UNCONTROLLED MIMO3 CASCADE NONLINEAR SYSTEM}

The uncontrolled MIMO3 cascade nonlinear system with three inputs, three outputs and $k+1=5000$ cascade connected subsystems is given in Figure 4. Each cascade is described with the next set of equations:

$$
\begin{aligned}
& x_{1}^{(i+1)}=f_{1}^{(i+1)}\left(x_{1}^{(i)}, \quad x_{2}^{(i)}, \quad x_{3}^{(i)}\right)=x_{2}^{(i)} \\
& x_{2}^{(i+1)}=f_{2}^{(i+1)}\left(x_{1}^{(i)}, \quad x_{2}^{(i)}, \quad x_{3}^{(i)}\right)=x_{3}^{(i)} \\
& x_{3}^{(i+1)}=f_{3}^{(i+1)}\left(x_{1}^{(i)}, \quad x_{2}^{(i)}, \quad x_{3}^{(i)}\right)=-l_{1} x_{3}^{(i)}-l_{2} x_{2}^{(i)}-l_{3} x_{1}^{(i)} \\
& l_{1}=-\left(x_{1}^{(i)}\right)^{2}+\frac{25}{16}, \quad l_{2}=0.5, \quad l_{3}=\text { const }, \quad i=\overline{0, \ldots, \quad 4999}
\end{aligned}
$$

The inputs to the first cascade are $x_{1}^{(0)}=0, x_{2}^{(0)}=0, x_{3}^{(0)}=0.17$ and $l_{3}$ is a variable parameter. Determination of the region where multiple spatial limit sets and chaos appear can be performed easily using bifurcation diagram. Firstly, based on equation (4), the values interval of the parameter $l_{3}$ is simply determined in the following way:

$$
\left|l_{3}\right| \leq\left(\begin{array}{l}
3 \\
3
\end{array}\right) \text {, i.e. }\left|l_{3}\right| \leq 1
$$

Then using spatial phase portraits, a subinterval of the interval $\left|l_{3}\right| \leq 1$ is determined for which the analyzed system, equation (7), is not unstable. In this way the precise narrow interval of the parameter $l_{3} \in[-0.4284,0.6256]$ is obtained. For this interval simulation of bifurcation diagram, Fig. 5, is performed using the Matlab M-file given in the Appendix. On the bifurcation diagram all three outputs of the last cascade $\mathbf{x}^{(5000)}=$ $\left[x_{1}^{5000)}, x_{2}^{(5000)}, \ldots, x_{3}^{(5000)}\right]$ are shown together as a function of variable parameter $l_{3}$. 


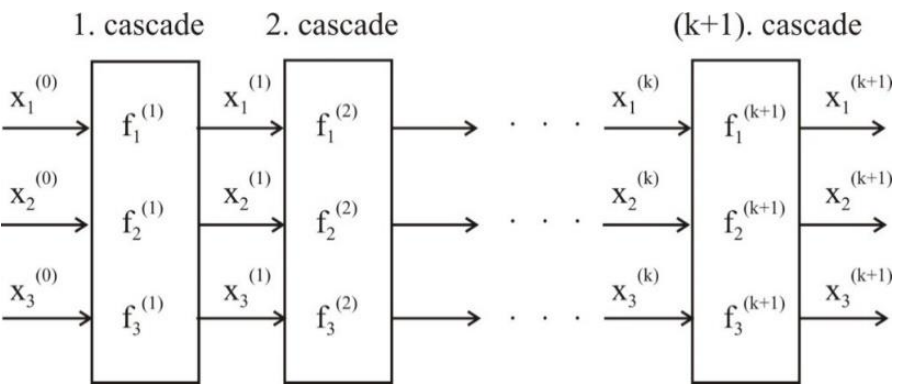

Fig. 4 Uncontrolled MIMO3 cascade nonlinear system

For $l_{3} \in[-0.4284,-0.4]$, Fig. 5 , the system is in the region of spatial chaos which is shown in the spatial phase portrait, Fig. $6 \mathrm{a}, l_{3}=-0.405$. For $l_{3} \in(-0.4,-0.25]$ one spatial limit set is formed. This is shown in the spatial phase portrait, Fig. $6 \mathrm{~b}$, for $l_{3}=-0.3$. One branch in bifurcation diagram for $l_{3} \in[-0.239,-0.07]$ corresponds to one stable focus, Fig. $6 \mathrm{c}, l_{3}=-0.2$. Two branches in the bifurcation diagram for $l_{3} \in(-0.07,0.18]$ correspond to two stable focuses, Fig. $6 \mathrm{~d}, l_{3}=0.1$. For $l_{3} \in(0.18,0.615)$ there exist two spatial limit sets, Fig. 6e, $l_{3}=0.3$. For $l_{3} \in[0.615,0.625]$ spatial chaos appears, Fig. 6f, $l_{3}=0.62$ and for $l_{3}>0.625$ the system is unstable.

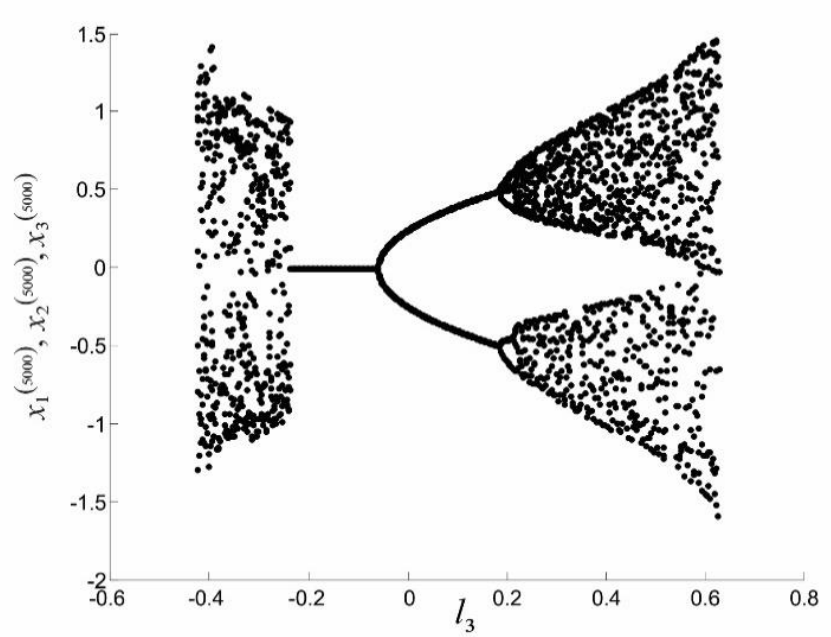

Fig. 5 Bifurcation diagram of uncontrolled MIMO3 system, (7) 

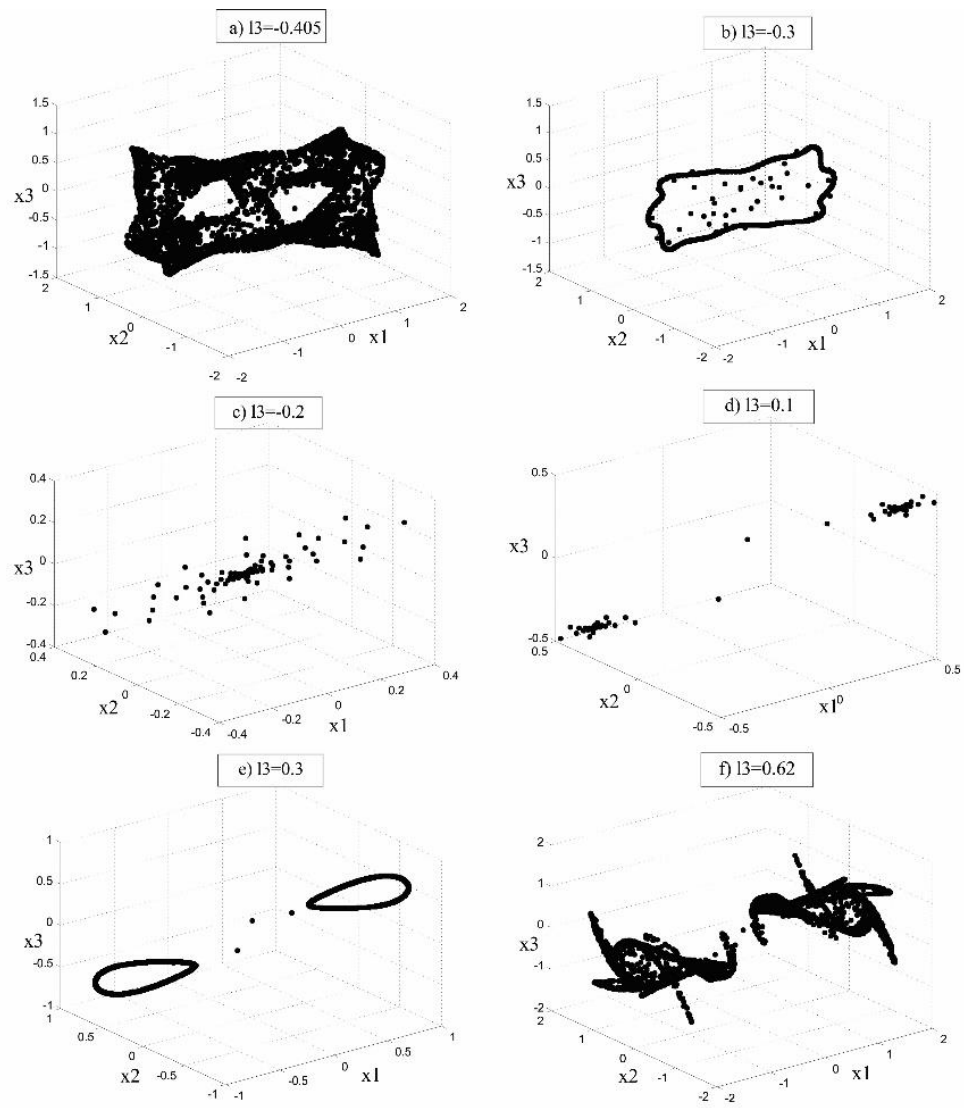

Fig. 6 Spatial phase portraits of uncontrolled MIMO3 system, (7)

\section{EXAMPLE OF CONTROLlED MIMO3 CASCADE NONLINEAR SySTEM}

In this Section, the modified Pyragas method of control is applied to the system (7). A controlled MIMO3 cascade nonlinear system is given in Figure 7. Each cascade of this MIMO3 controlled system is given by:

$$
\begin{aligned}
& y_{1}^{(i+1)}=f_{1}^{(i+1)}\left(y_{1}^{(i)}, \quad y_{2}^{(i)}, \quad y_{3}^{(i)}\right)=y_{2}^{(i)} \\
& y_{2}^{(i+1)}=f_{2}^{(i+1)}\left(y_{1}^{(i)}, \quad y_{2}^{(i)}, \quad y_{3}^{(i)}\right)=y_{3}^{(i)} \\
& y_{3}^{(i+1)}=f_{3}^{(i+1)}\left(y_{1}^{(i)}, \quad y_{2}^{(i)}, \quad y_{3}^{(i)}\right)-u^{(i)}= \\
& =-l_{1} y_{3}^{(i)}-l_{2} y_{2}^{(i)}-l_{3} y_{1}^{(i)}-u^{(i)} \\
& u^{(i)}=K\left(y_{3}^{(i)}-y_{2}^{(i)}\right), \quad i=\overline{0,4999} \\
& l_{1}=-\left(y_{1}^{(i)}\right)^{2}+\frac{25}{16}, \quad l_{2}=0.5, \quad l_{3}=\text { const, } i=\overline{0, \ldots, 4999}
\end{aligned}
$$


Inputs to the first cascade of the controlled MIMO3 cascade system (9), $\mathbf{y}^{(0)}=$ $\left[y_{1}{ }^{(0)}, y_{2}{ }^{(0)}, y_{3}{ }^{(0)}\right]$, and inputs to the first cascade of the uncontrolled MIMO3 cascade system (7), $\mathbf{x}^{(0)}=\left[x_{1}{ }^{(0)}, x_{2}{ }^{(0)}, x_{3}{ }^{(0)}\right]$, are the same, i.e., $y_{1}{ }^{(0)}=0, y_{2}{ }^{(0)}=0, y_{3}{ }^{(0)}=0.17$. In equations (9) $K$ is the control parameter and $l_{3}$ is the variable parameter.

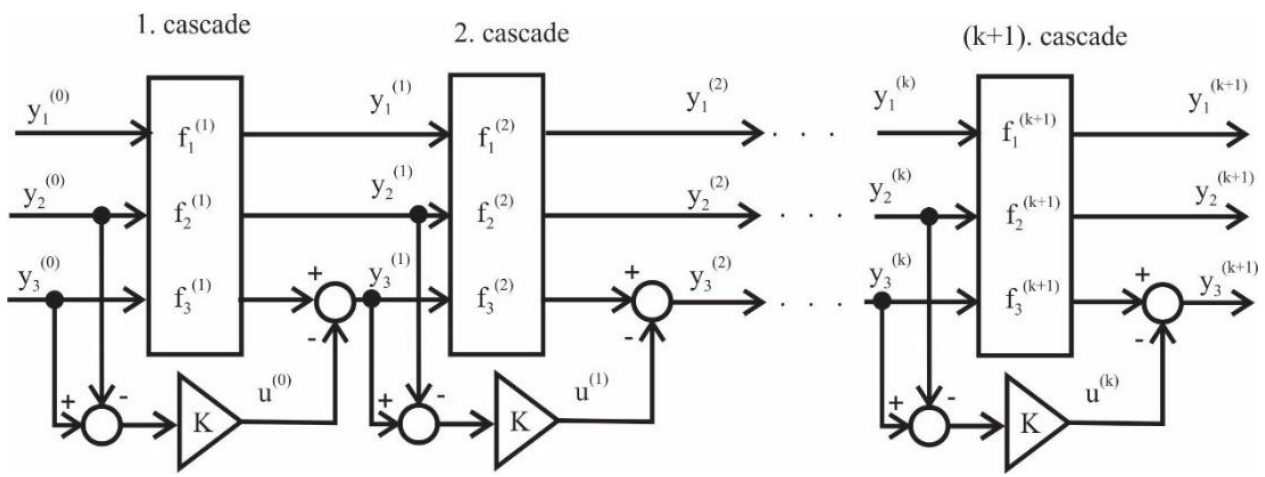

Fig. 7 Controlled MIMO3 cascade nonlinear system

For $K=-0.1$ a simulation of a bifurcation diagram is performed, Fig. 8. This diagram is obtained using the Matlab M-file given in the Appendix. On the bifurcation diagram all three outputs of the last cascade $\mathbf{y}^{(5000)}=\left[y_{1}^{(5000)}, y_{2}^{(5000)}, y_{3}^{(5000)}\right]$ are shown together as a function of the variable parameter $l_{3}$. It can be seen that the interval of the variable parameter $l_{3}$ is changed in comparison with the same interval for the uncontrolled MIMO3 system (7). Namely, it is extended on the right side, i.e., $l_{3} \in[-0.428,0.91]$. Comparing the bifurcation diagrams given in Figs. 5 and 8 (and also the spatial phase portraits in Figs. 6 and 9) we can conclude:

1) Region of one stable focus of the controlled MIMO3 system (9) is extended in relation to the same region of the uncontrolled MIMO3 system (7). Namely, this region includes both the region of one stable focus $l_{3} \in[-0.22,-0.07]$ given in Fig.5, and the region of two stable focuses $l_{3} \in(-0.07,0.137]$ given in the same figure.

2) Region of two spatial limit sets of the uncontrolled MIMO3 system for $l_{3} \in(0.18,0.57)$ has become the region of two stable focuses in the case of the controlled MIMO3 system.

3) Region of chaos and unstable region given in the bifurcation diagram, Fig.5, after using the modified Pyragas method of control, have become the region of two spatial limit sets, $l_{3} \in(0.615,0.91]$. 


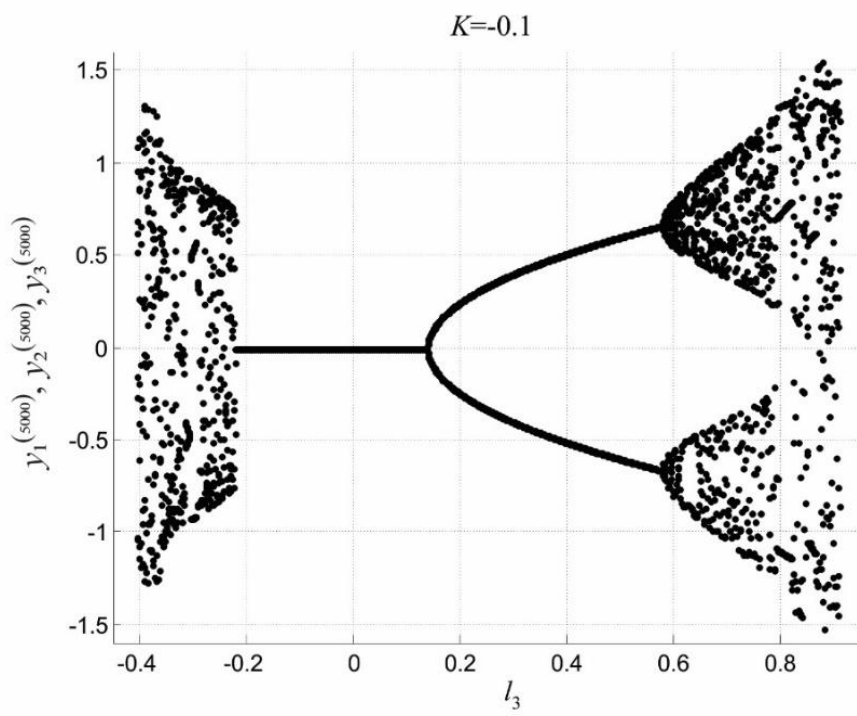

Fig. 8 Bifurcation diagram of controlled MIMO3 system, (9)

Spatial phase portraits of the controlled MIMO3 system, (9), are presented in Fig.9. Comparing the spatial phase portraits given in Fig. 9 with spatial phase portraits given on Fig. 6, we are coming to the same conclusions as well as by comparing the bifurcation diagrams given in Figs. 5 and 8. These conclusions are summarized in Table1.

Table1 Dynamics comparison of uncontrolled and controlled MIMO3 systems using Figs. 6 and 9

\begin{tabular}{lll}
\hline Parameter $l_{3}$ & Uncontrolled MIMO3 system & Controlled MIMO3 system \\
\hline$l_{3}=-0.405$ & chaos & chaos \\
$l_{3}=-0.3$ & spatial limit set & spatial limit set \\
$l_{3}=-0.2$ & one stable focus & one stable focus \\
$l_{3}=0.1$ & two stable focuses & one stable focus \\
$l_{3}=0.3$ & two spatial limit sets & two stable focuses \\
$l_{3}=0.62$ & chaos & two spatial limit sets \\
$l_{3}=0.85$ & system is unstable & two spatial limit sets \\
$l_{3}=0.91$ & system is unstable & chaos \\
\hline
\end{tabular}



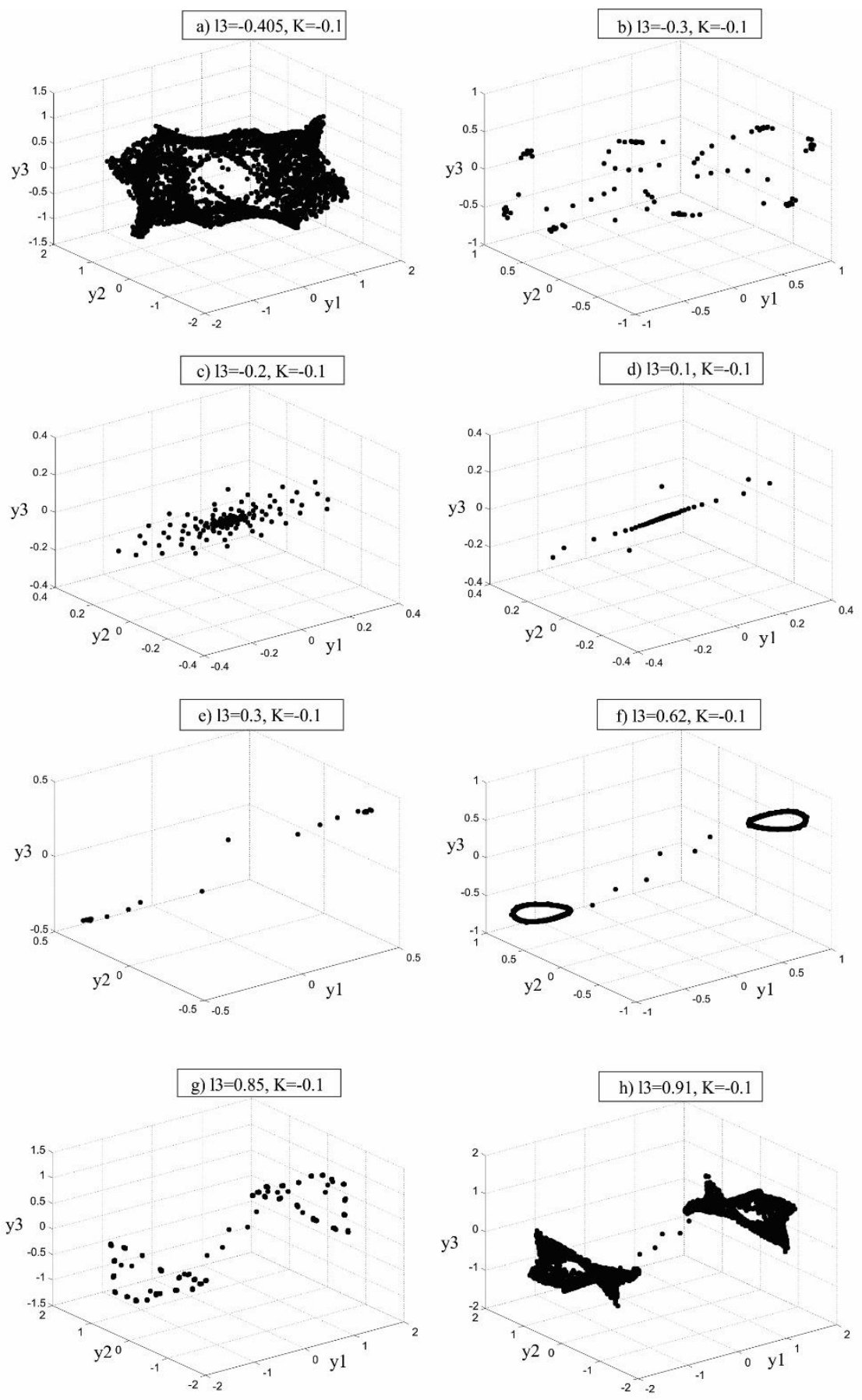

Fig. 9 Spatial phase portraits of controlled MIMO3 system, (9) 


\section{CONCLUSION}

The modified Pyragas method for multiple spatial limit sets and chaos control in MIMO cascade nonlinear systems is presented in this paper. Besides the application of spatial phase portraits for dynamics analysis of controlled and uncontrolled MIMO cascade nonlinear systems, [3], bifurcation diagrams are used in this paper. In the case of monitoring the dynamics of an uncontrolled MIMO system with a large number of cascades $(k+1)$, using bifurcation diagram, is based on monitoring all outputs of the last cascade $\mathbf{x}^{(k+1)}=\left[x_{1}^{(k+1)}, \ldots, x_{n}^{(k+1)}\right]$ as a function of the variable parameter $l_{n}$. Bifurcation diagram of a controlled MIMO system is based on monitoring all outputs of the last cascade $\mathbf{y}^{(k+1)}=\left[y_{1}^{(k+1)}, \ldots, y_{n}^{(k+1)}\right]$ as a function of the variable parameter $l_{n}$. These diagrams show the dynamics of both systems completely. This is confirmed by comparing the segments of bifurcation diagrams with the spatial phase portraits. By comparing the bifurcation diagram of a controlled MIMO3 system with the bifurcation diagram of an uncontrolled MIMO3 system, we can conclude that the stability region of the controlled system is expanded thanks to the application of the modified Pyragas method. The controlled system is also moved from the region of multiple spatial limit sets into a region of two stable focuses, and from the region of chaos in a region of two spatial limit sets.

\section{APPENDIX: MATLAB M-FILES}

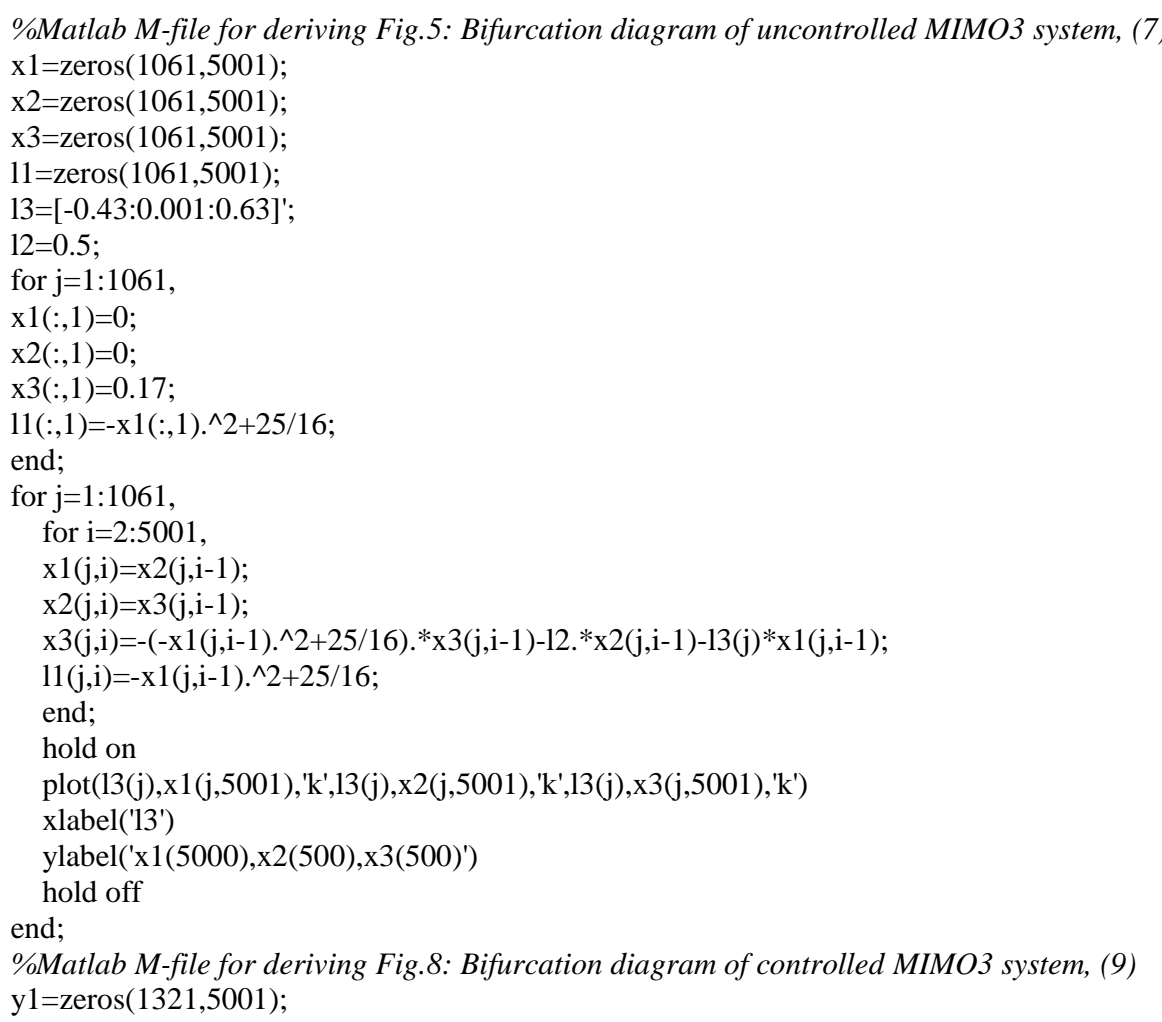




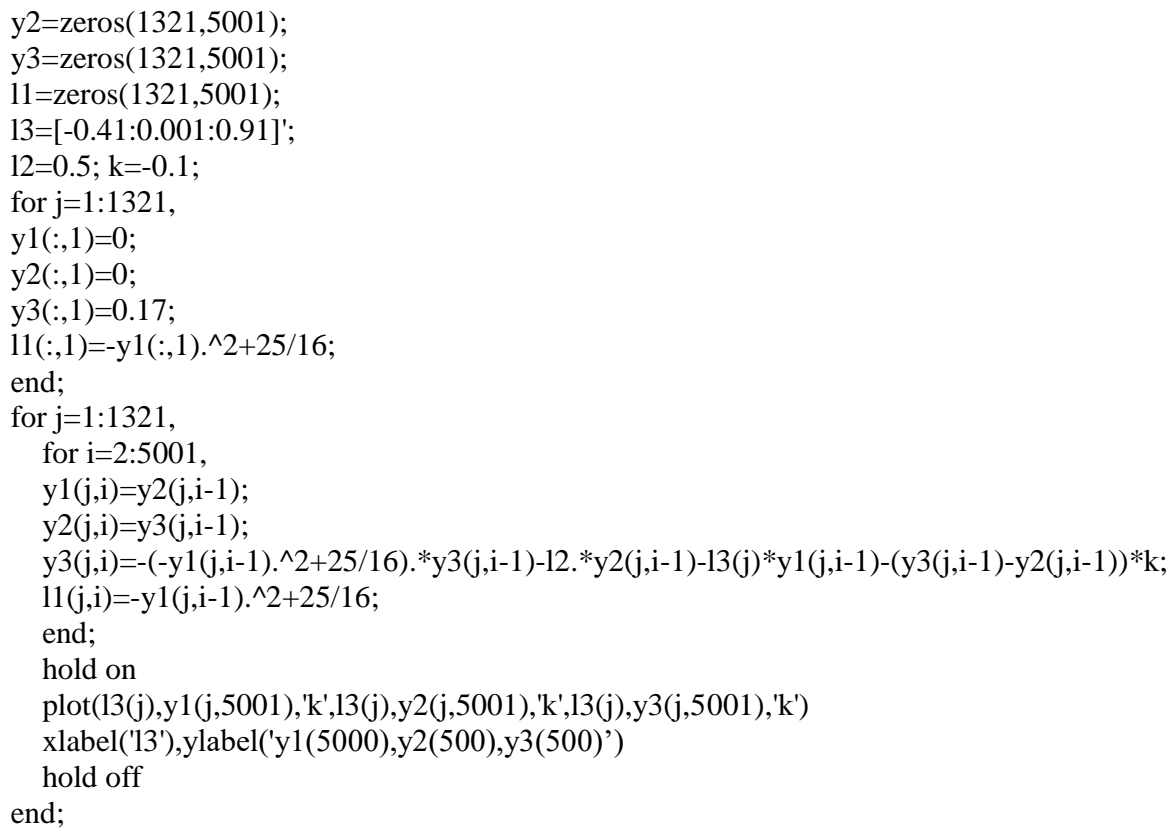

\section{REFERENCES}

[1] K. Pyragas, "Delayed feedback control of chaos," Philos. Trans. A Math. Phys. Eng. Sci., 364(1846), pp.2309-2334 (2006), doi:10.1098/rsta.2006.1827

[2] W. Perruquetti and J.P. Barbot, Chaos in Automatic Control, Taylor\&Francis (2005)

[3] Zlatkovic, B. M.; Samardzic, B.: Analysis and control of spatial limit sets and spatial chaos appearance in MIMO cascade connected nonlinear systems, Asian journal of control, Vol. 21, No.5 (2019), doi:10.1002/asjc. 1860 .

[4] K. T. Alligood, T. D. Sauer and J. A. Yorke, Chaos, An introduction to dynamical systems, Springer (1997).

[5] S.Lefschetz, Differential equations: Geometric theory, Dower Publications, New York (1990).

[6] P. Agathomeis, E.I. Jury and M. Mansour, "Criteria for Absence of Limit Cycles in Two-dimensional Discrete Systems," IEEE Trans. ONASSP, Vol. 32, No. 2, pp. $432-434$ (1984).

[7] B. Dankovic, M. Stankovic and B. Vidojkovic, "Simulation of convergent, oscillatory and chaotic dynamics of cascade systems," The 7th Symposium of Mathematics and its Applications, Proceedings, Timisoara, pp. 101-106 (1997).

[8] B. Dankovic, B. Vidojkovic and B. M. Vidojkovic, "On the chaos in cascade systems for rubber strip transportation," The Fourth International Conference, Heavy Machinery, Proceedings, HM 2002, Serbia, pp. A.97 - A 100 (2002)

[9] B. Samardzic and B. M. Zlatkovic, "Simulation of bifurcation and escape-time diagrams of cascadeconnected nonlinear systems for rubber strip transportation," Nonlinear Dynamics, Vol 67, No. 2, pp. 1105 - 1113 (2012), doi:10.1007/s11071-011-0054-y.

[10] B. Samardzic and B. M. Zlatkovic, "Analysis of spatial chaos appearance in cascade connected nonlinear electrical circuits," Chaos, solitons and fractals: the interdisciplinary journal of Nonlinear Science, and Nonequilibrium and Complex Phenomena, Vol. 95, pp. 14-20 (2017), doi: 10.1016/j.chaos.2016.12.003.

[11] B. M. Zlatkovic and B. Samardzic, "One way for the probability of stability estimation of discrete systems with randomly chosen parameters," IMA Journal of Mathematical Control and Information, Vol. 29, No. 3, pp. 329-341 (2012), doi.org/10.1093/imamci/dnr041. 